\title{
Structural Determinants of Tissue Tropism and In Vivo Pathogenicity for the Parvovirus Minute Virus of Mice
}

\author{
Maria Kontou, ${ }^{1} \dagger$ Lakshmanan Govindasamy, ${ }^{1}$ Hyun-Joo Nam, ${ }^{1}$ Nathan Bryant, ${ }^{1}$ \\ Antonio L. Llamas-Saiz ${ }^{2}+$ Concepción Foces-Foces, ${ }^{2}$ Eva Hernando, ${ }^{3} \S$ \\ Mari-Paz Rubio, ${ }^{3}$ I Robert McKenna, ${ }^{1}$ José M. Almendral, ${ }^{3}$ \\ and Mavis Agbandje-McKenna ${ }^{1 *}$ \\ Department of Biochemistry and Molecular Biology, College of Medicine, University of Florida, \\ Gainesville, Florida 32610-0245, ${ }^{1}$ and Departamento de Cristalografia, Instituto de \\ Quimica-Fisica "Rocasolano," CSIC, Serrano 119, 28006 Madrid, ${ }^{2}$ and \\ Centro de Biologia Molecular "Severo Ochoa" (UAM-CSIC), \\ Universidad Autónoma de Madrid, Cantoblanco, \\ 28049 Madrid, ${ }^{3}$ Spain
}

Received 11 March 2005/Accepted 25 May 2005

\begin{abstract}
Two strains of the parvovirus minute virus of mice (MVM), the immunosuppressive (MVMi) and the prototype (MVMp) strains, display disparate in vitro tropism and in vivo pathogenicity. We report the crystal structures of MVMp virus-like particles $\left(M V M p_{b}\right)$ and native wild-type (wt) empty capsids $\left(M_{V M M p}\right)$, determined and refined to 3.25 and $3.75 \AA$ resolution, respectively, and their comparison to the structure of MVMi, also refined to $3.5 \AA$ resolution in this study. A comparison of the $\mathrm{MVMp}_{\mathrm{b}}$ and $M V M p_{\mathrm{e}}$ capsids showed their structures to be the same, providing structural verification that some heterologously expressed parvovirus capsids are indistinguishable from wt capsids produced in host cells. The structures of MVMi and MVMp capsids were almost identical, but local surface conformational differences clustered from symmetry-related capsid proteins at three specific domains: (i) the icosahedral fivefold axis, (ii) the "shoulder" of the protrusion at the icosahedral threefold axis, and (iii) the area surrounding the depression at the icosahedral twofold axis. The latter two domains contain important determinants of MVM in vitro tropism (residues 317 and 321 ) and forward mutation residues (residues 399, 460, 553, and 558) conferring fibrotropism on MVMi. Furthermore, these structural differences between the MVM strains colocalize with tropism and pathogenicity determinants mapped for other autonomous parvovirus capsids, highlighting the importance of common parvovirus capsid regions in the control of virus-host interactions.
\end{abstract}

Viral tissue tropism and pathogenesis are highly dependent on well-orchestrated and defined interactions that occur between the viral pathogen and its host. The single-stranded Parvoviridae infect a broad range of natural hosts that include invertebrates and mammals, and pathogenic members cause serious disease in the young and immunocompromised adults. However, disparities in tissue tropism and in vivo pathogenicity can be observed between highly homologous strains for several members of the Parvoviridae. The combination of molecular analysis with disease outcomes for a number of strains of the Aleutian mink disease parvovirus (AMDV) $(9,10)$, canine parvovirus (CPV), and feline panleukopenia virus (FPV) (46, $61,62)$, and for porcine parvovirus (PPV) $(8,41)$, has provided

\footnotetext{
* Corresponding author. Mailing address: Department of Biochemistry and Molecular Biology, College of Medicine, University of Florida, Gainesville, FL 32610-0245. Phone: (352) 392-5694. Fax: (352) 392-3422. E-mail: mckenna@ufl.edu.

$\dagger$ Present address: University of Thessaly, Argonafton and Filellinon, 38221 Volos, Greece.

\$ Present address: Unidade de Raios X. RIAIDT, Vicerrectorado de Investigación, Universidade de Santiago de Compostela, E-15706, Santiago de Compostela, Spain.

$\S$ Present address: Department of Pathology, Memorial Sloan-Kettering Cancer Center, New York, NY 10021.

I Present address: Instituto de Biomedicina de Valencia (CSIC), 46010 Valencia, Spain
}

models for probing the determinants of parvovirus tissue tropism and pathogenicity. In the parvovirus minute virus of mice (MVM) model adopted in this study, the prototype strain (MVMp) replicates in vitro in mouse fibroblast cell lines, while the immunosuppressive strain (MVMi) replicates in mouse $\mathrm{T}$ lymphocytes and mouse hematopoietic precursors. In vivo, MVMp infection of newborn mice is asymptomatic, while MVMi infection of newborn mice as well as of adult mice with severe combined immunodeficiency syndrome (SCID) is lethal $(11,12,47,51,52)$.

Parvovirus capsids are $\sim 260 \AA$ in diameter and contain 60 copies (in total) of viral protein 1 (VP1) to $\mathrm{VP} 4$, in a $\mathrm{T}=1$ icosahedral capsid arrangement, with the smallest VP, depending on the virus member, being the major capsid viral protein. The number of capsid VP species per virion differs among parvoviruses. For example, the AMDV capsid contains only two polypeptides, VP1 and VP2; MVM has three, VP1 to VP3; and members of the subfamily Densovirinae have four, VP1 to VP4. The VPs are overlapping, with the entire sequence of VP4 contained within VP3; VP3 is contained in VP2, which is in turn contained within VP1; VP1 has a unique N-terminal domain (58). The VPs are translated from the same mRNA or result from posttranslational cleavage. For example, in MVM, VP3 is formed by postassembly cleavage of approximately 18 to 20 amino acids from the $\mathrm{N}$ terminus of VP2 in full (DNAcontaining) infectious virions. This cleavage event is not seen 


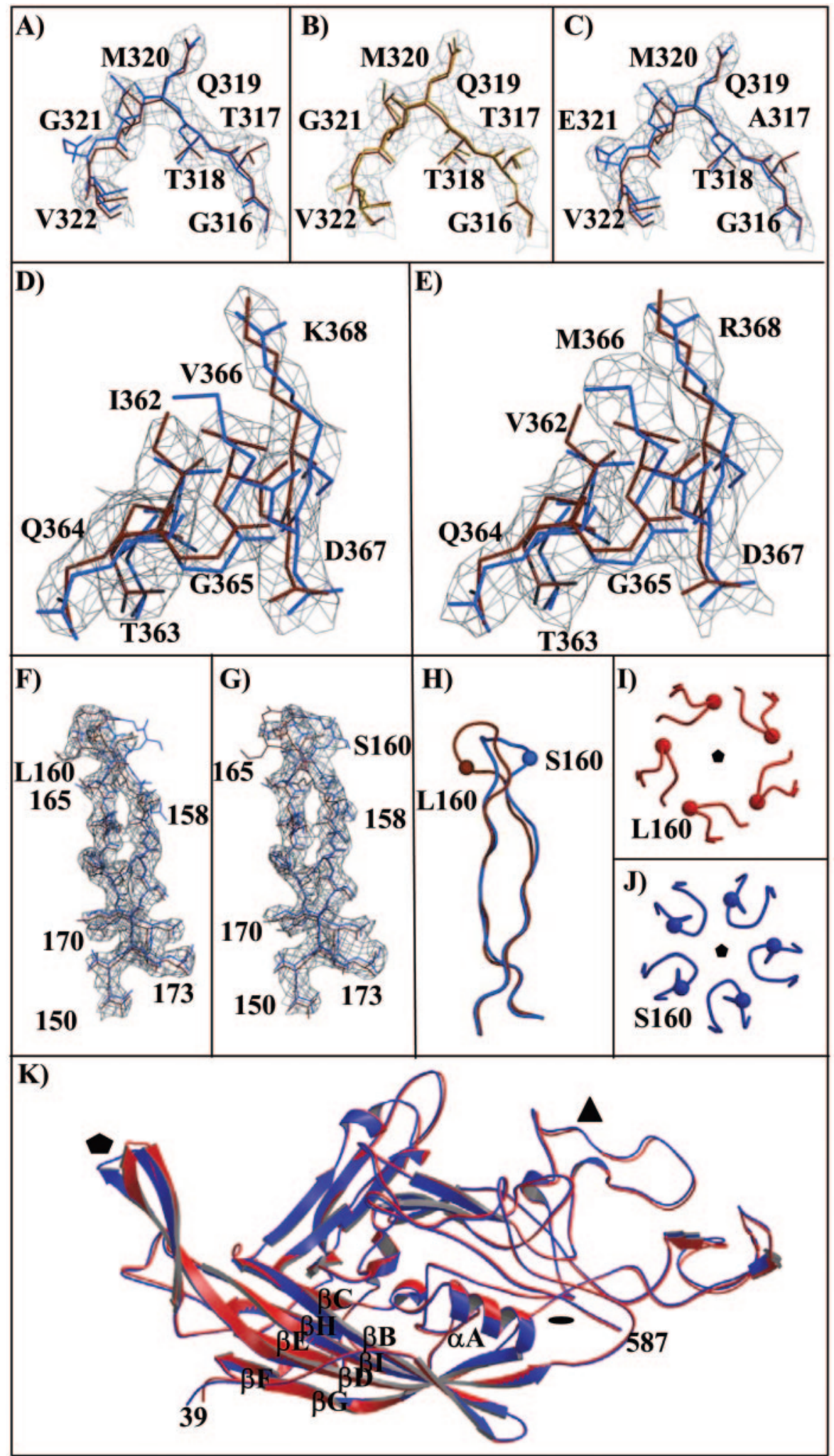

FIG. 1. Structure of MVM VP2. The MVMp $\mathrm{p}_{\mathrm{b}}$ structure is shown in red, that of MVMp is in brown, and that of MVMi is in blue. (A to C) Electron density $\left(2 \mathrm{~F}_{\mathrm{o}}-\mathrm{F}_{\mathrm{c}}\right.$ ) maps (gray wire) for $\mathrm{MVMp}$, $\mathrm{MVMp}_{\mathrm{e}}$, and MVMi amino acids 316 to 322, respectively, containing MVMi/p differences at positions 317 and 321, the allotropic determinants. The MVMp and MVMi structures are superimposed in panels $\mathrm{A}$ and $\mathrm{C}$, and those of $\mathrm{MVMp}_{\mathrm{b}}$ and $\mathrm{MVMp}_{\mathrm{e}}$ are superimposed in panel B. (D and E) Electron density $\left(2 \mathrm{~F}_{\mathrm{o}}-\mathrm{F}_{\mathrm{c}}\right.$ ) maps (gray wire) for MVMp $\mathrm{p}_{\mathrm{b}}$ and $\mathrm{MVMi}$ amino acids 362 to 368 , respectively, containing $\mathrm{MVMi} / \mathrm{p}$ differences at positions 362,366 , and 368 . (F and $\mathrm{G})$ Electron density $\left(2 \mathrm{~F}_{\mathrm{o}}-\mathrm{F}_{\mathrm{c}}\right.$ ) maps $(\mathrm{gray}$ wire) for $\mathrm{MVMp}_{\mathrm{b}}$ and MVMi amino acids 150 to 173, respectively, containing an MVMi/p difference at position 160. (H) Superimposition of coil representations for the amino acid stretch shown in panels $\mathrm{F}$ and $\mathrm{G}$ for $\mathrm{MVMp}_{\mathrm{b}}$ (red) and MVMi (blue). The orientations of the structures in 
in empty (no DNA) particles digested in vitro with trypsin, unless exposure of VP2 is induced by heat $(19,27)$. The molecular sizes of the MVM proteins are 83,000, 64,000, and 61,000 Da for VP1, VP2, and VP3, respectively.

The 3-dimensional structures of several parvoviruses, adenoassociated virus serotype 2 (AAV2), human parvovirus B19 (B19), CPV, Galleria mellonella densovirus (GmDNV), FPV, MVMi, and PPV, have been determined using X-ray crystallography $(1-3,33,54,55,63,67,68)$. Crystal structures of host range and antigenic mutants of CPV, as well as of CPV and FPV under various $\mathrm{pH}$ and ionic conditions, have also been determined $(26,36,53)$. The structures of AMDV strain $G$ (AMDV-G), AAV2, AAV4, AAV5, B19-globoside receptor complex, CPV-Fab complex, and Junonia coenia densovirus $(J c \mathrm{DNV})$ have been determined by cryoelectron microscopy and image reconstruction $(13,18,34,39,44,64,65)$. The parvovirus capsid VP topology is highly conserved, even for members that are only $\sim 20 \%$ identical at the amino acid sequence level, such as AAV2 and B19 (33), with variations localized to surface loop regions between strands of a core $\beta$-barrel domain. The characteristic parvovirus capsid surface features include protrusions at or surrounding the icosahedral threefold axes and depressions at the icosahedral twofold axes and around the fivefold axes. Exceptions to this general surface topology are seen in the smoother $G m \mathrm{DNV}$ and $J c \mathrm{DNV}$ capsids as a result of smaller loop insertions between their $\beta$-strands $(13,54)$.

Structural mapping of amino acids reported to control tissue tropism and pathogenicity onto the capsid proteins of AMDV-G, CPV, FPV, MVMi, and PPV localize these regions on or close to variable capsid surface loops $(2,3,6,8,9,17,24$, $26,28,30,31,38,45,53,55,61,62,69)$. In a continued effort to elucidate the role of the parvovirus capsid structure in tropism and pathogenicity by utilizing the MVM model, we report the structures of native wild-type (wt) empty capsids of MVMp containing VP1 and VP2 $\left(\mathrm{MVMp}_{\mathrm{e}}\right)$ and of baculovirus-expressed VP2 virus-like capsids (VLPs) $\left(\mathrm{MVMp}_{\mathrm{b}}\right)$ determined and refined to 3.75 and $3.25 \AA$ resolution, respectively. The $\mathrm{MVMp}_{\mathrm{b}}$ structure is further compared to that of MVMi (3), also refined in the present study. We observe local surface conformational differences between the capsids of the MVM strains occurring at the icosahedral fivefold axis, on the "shoulder" of protrusions at the icosahedral threefold axis, and in and surrounding the depression at the icosahedral twofold axis. Comparison of the MVM VP2 structures to those of CPV, FPV, and PPV identified similar hot spots of surface loop structure variations, some of which colocalize with CPV/FPV, PPV, and ADV strain tissue tropism and pathogenicity determinants. These observations suggest the common utilization of variable parvovirus capsid regions by highly homologous viral strains with different tropisms and pathogenicities.
TABLE 1. Data collection and processing statistics

\begin{tabular}{llll}
\hline \multicolumn{1}{c}{ Parameter } & \multicolumn{1}{c}{ MVMp $_{\mathrm{b}}$} & \multicolumn{1}{c}{ MVMp $_{\mathrm{e}}$} & \multicolumn{1}{c}{ MVMi } \\
\hline Resolution $(\AA)$ & $20-3.25$ & $20-3.75$ & $20-3.5$ \\
Space group & $\mathrm{C} 2$ & $\mathrm{C} 2$ & $\mathrm{C} 2$ \\
Completeness $(\%)$ & 64.2 & 35.4 & 63.7 \\
$R_{\text {merge }}(\%)^{a}$ & 16.2 & 16.5 & 15.5 \\
Total no. of reflections $^{\circ}$ & 557,706 & 200,083 & 441,933 \\
Unit cell parameters $\left(\AA{ }^{\circ}\right)$ & $a=448.7$ & $a=448.7$ & $a=448.7$ \\
& $b=416.5$ & $b=416.5$ & $b=416.7$ \\
& $c=306.1$ & $c=306.1$ & $c=305.3$ \\
& $\beta=95.9$ & $\beta=95.9$ & $\beta=95.8$ \\
\hline
\end{tabular}

${ }^{a}$ Calculated as $\Sigma_{h} \Sigma_{i}\left|I_{h, i}-\left(I_{h}\right)\right| \Sigma_{h} \Sigma_{i}\left(I_{h, i}\right) \times 100$, where $h$ refers to unique reflection indices and $i$ indicates symmetry equivalent indices.

\section{MATERIALS AND METHODS}

Virus and VLP production and crystallization. $\mathrm{MVMp}_{\mathrm{e}}, \mathrm{MVMp}_{\mathrm{b}}$, and empty MVMi capsids were produced and purified as previously described (27, 37). The crystallization and preliminary characterization of the X-ray diffraction data for $\mathrm{MVMp}_{\mathrm{b}}$ have been reported elsewhere (27). $\mathrm{MVMp}_{\mathrm{e}}$ crystals were grown under the same conditions used for $\mathrm{MVMp}_{\mathrm{b}}(27)$, with the virus at a concentration of $10 \mathrm{mg} / \mathrm{ml}$ in $10 \mathrm{mM}$ Tris- $\mathrm{HCl}(\mathrm{pH} 7.5)-8 \mathrm{mM} \mathrm{CaCl}_{2}$, and with 0.5 to $1.0 \%$ polyethylene glycol 8000 as a precipitant.

Data collection, indexing, and processing. Data on $\mathrm{MVMp}_{\mathrm{b}}$ and $\mathrm{MVMp}_{\mathrm{e}}$ crystals were collected at $4^{\circ} \mathrm{C}$ on a MAR30 image plate and on an ADSC Quantum 4 CCD detector at beamline 9.6 at the Daresbury Synchrotron Radiation Source (United Kingdom), operating at a wavelength $(\lambda)$ of $0.870 \AA$, and on a MAR30 image plate detector at the EMBL X31 beamline at DESY (Hamburg, Germany), operating at a $\lambda$ of $1.071 \AA$ A. Oscillation images were indexed and processed with the DENZO program (43) and were scaled, merged, and postrefined using SCALEPACK (43). The space groups for the crystals were monoclinic $\mathrm{C} 2$, with postrefined cell parameters (Table 1) that were pseudoisomorphous to that reported for MVMi $(a=448.7 \AA, b=416.7 \AA, c=305.3$ $\AA$, and $\left.\beta=95.8^{\circ}\right)(37)$. The data processing and scaling of images from a total of 30 crystals for $\mathrm{MVMp}_{\mathrm{b}}$ and 26 crystals for $\mathrm{MVMp}_{\mathrm{e}}$ followed procedures utilized for MVMi (37). The data collection and processing statistics are summarized in Table 1.

Structure determination. The capsid structures of $\mathrm{MVMp}_{\mathrm{b}}$ and $\mathrm{MVMp}_{\mathrm{e}}$ were determined by molecular replacement (50), involving the determination of particle orientations and positions within the crystal unit cell. The $\mathrm{MVMp}_{\mathrm{b}}$ and $\mathrm{MVMp}_{\mathrm{e}} \mathrm{C} 2$ cells contained two half-particles with different orientations in the asymmetric unit as previously described for MVMi (37). The orientations of the two half-particles were determined initially by a self-rotation function (59) computed using $\sim 11 \%$ of the data in the 10 to $5 \AA$ resolution range as large terms to represent the second Patterson function and then, more accurately, by the locked self-rotation function (59) computed using $\sim 16 \%$ of the data in the 4 to $3.25 \AA$ resolution range for $\mathrm{MVMp}_{\mathrm{b}}$ and $\sim 16 \%$ in the 5 to $3.75 \AA$ resolution range for $\mathrm{MVMp}_{\mathrm{e}}$. Packing considerations suggested that the centers of the complete $\mathrm{T}=1 \mathrm{MVMp}$ particles are located on the crystallographic twofold axes at $(0,0,0)$ (particle 1$)$ and $(0, \sim 1 / 2,1 / 2)$ (particle 2 ), as was also reported for MVMi (37). Molecular replacement calculations, applying 60-fold noncrystallographic symmetry (NCS), were performed using the Purdue suite of programs (50), with the structure of CPV as the initial phasing model, for data in the 20 to 3.25 and 20 to $3.75 \AA$ resolution ranges for $\mathrm{MVMp}_{\mathrm{b}}$ and $\mathrm{MVMp}_{\mathrm{e}}$, respectively. The inner and outer radii of the particles were set at 70 and $145 \AA$, respectively, to define solvent boundaries. The calculations were performed in an artificial "H-cell," a unit cell containing a single particle in a known standard orientation, as defined by Rossmann et al. in 1992 (50), and were utilized as described in the structure determination of MVMi (37). The MVMi structure (3) was not con-

panels $\mathrm{F}$ to $\mathrm{H}$ are perpendicular to the icosahedral fivefold axis. (I and J) Coil representations for residues 157 to 164 , with altered conformations in $\mathrm{MVMp}_{\mathrm{b}}$ and $\mathrm{MVMi}$, respectively. The approximate fivefold axis is shown in the filled pentagon. (K) Superimposition of the ribbon diagrams of $\mathrm{MVMp}_{\mathrm{b}}$ (red) and MVMi (blue) VP2, illustrating $\beta$-strand, helical, and loop regions. Conserved $\beta$-strands $\beta \mathrm{B}$ to $\beta \mathrm{I}$, helix $\alpha \mathrm{A}$, and residues 39 and 587, the first $\mathrm{N}$-terminal residue modeled and the C-terminal residue, respectively, are labeled. The approximate icosahedral twofold (filled oval), threefold (filled triangle), and fivefold (filled pentagon) axes are shown. This figure was generated with the BOBSCRIPT (22) (A to J) and PyMol (21) (K) programs. 
TABLE 2. Refinement statistics for $\mathrm{MVMp}_{\mathrm{b}}, \mathrm{MVMp}_{\mathrm{e}}$, and MVMi

\begin{tabular}{|c|c|c|c|}
\hline Parameter & $\mathrm{MVMp}_{\mathrm{b}}$ & $\mathrm{MVMp}_{\mathrm{e}}$ & MVMi \\
\hline Resolution $(\AA)$ & $20-3.25$ & $20-3.75$ & $20-3.5$ \\
\hline Space group/crystal system & $\mathrm{C} 2 /$ monoclinic & $\mathrm{C} 2 /$ monoclinic & $\mathrm{C} 2 /$ monoclinic \\
\hline$R_{\mathrm{factor}}(\%)^{a}$ & 29.85 & 32.35 & 32.55 \\
\hline$R_{\text {free }}(\%)^{b}$ & 30.62 & 32.95 & 32.93 \\
\hline Molecular averaging NCS correlation coefficient & 0.8765 & 0.9078 & 0.8615 \\
\hline No. of reflections & 557,706 & 200,083 & 441,933 \\
\hline No. of independent reflections & 517,599 & 190,120 & 437,558 \\
\hline No. of protein atoms & 4,317 & 4,317 & 4,328 \\
\hline No. of solvent molecules & 121 & 47 & 101 \\
\hline RMSD, bond lengths $(\AA)$ & 0.010 & 0.013 & 0.010 \\
\hline RMSD, bond angles $\left({ }^{\circ}\right)$ & 1.583 & 1.718 & 1.619 \\
\hline Avg B factor, main chain $\left(\AA^{2}\right)$ & 19.29 & 20.58 & 20.91 \\
\hline Avg B factor, side chain $\left(\AA^{2}\right)$ & 20.65 & 20.85 & 22.05 \\
\hline Avg B factor for protein/water $\left(\AA^{2}\right)$ & $20.86 / 52.98$ & $20.71 / 34.510$ & $21.47 / 47.61$ \\
\hline Residues in the most/additional/generously allowed regions (\%) & $81.2 / 18.8 / 0$ & 79.1/8.8/10.0 & $70.9 / 26.0 / 3.2$ \\
\hline
\end{tabular}

${ }^{a}$ Calculated as $\Sigma|| \mathrm{F}_{\mathrm{o}}|-| \mathrm{F}_{\mathrm{c}}|\Sigma| \mathrm{F}_{\mathrm{o}} \mid \times 100$, where $\mathrm{F}_{\mathrm{o}}$ and $\mathrm{F}_{\mathrm{c}}$ are the observed and calculated structure factors, respectively.

${ }^{b}$ Same as $R_{\text {factor }}$, but calculated with a $5 \%$ randomly selected fraction of the reflection data not included in the refinement.

sidered as a starting model to avoid phase bias due to the high degree of identity ( $97 \%$ ) between the amino acid sequences of MVMi and MVMp. The "climb" procedure of the ENVELOPE program (50) was used to refine the orientations and positions of the particles several times during phase refinement cycles.

After 75 cycles of molecular replacement calculations, the final orientations $(\psi, \phi$, and $\kappa)$ for the two half-particles were $\left(0,0,29.83^{\circ}\right)$ and $\left(0,0,-106.42^{\circ}\right)$ for $\mathrm{MVMp}_{\mathrm{b}}$ and $\left(0,0,30.06^{\circ}\right)$ and $\left(0,0,-106.78^{\circ}\right)$ for $\mathrm{MVMp}_{\mathrm{e}}$. The final particle positions (in fractional coordinates) were $(0,0,0)$ and $(0,0.49985,0.50000)$ for $\mathrm{MVMp}_{\mathrm{b}}$ and $(0,0,0)$ and $(0,0.50000,0.50000)$ for $\mathrm{MVMp}_{\mathrm{e}}$. The final averaging correlation coefficients were 0.74 and 0.71 for $\mathrm{MVMp}_{\mathrm{b}}$ and $\mathrm{MVMp}_{\mathrm{e}}$, respectively. Residues 39 to 587 were built into the $\mathrm{H}$-cell electron density map. The resulting model coordinates were transformed to the crystallographic unit cell by using the $[\mathrm{P}]$ matrix, which defines a rotational relationship between the structure in the $\mathrm{H}$-cell and the reference particle in the crystallographic unit cell (50). These models were used for all subsequent refinement steps of the $\mathrm{MVMp}_{\mathrm{b}}$ and $\mathrm{MVMp}_{\mathrm{e}}$ structures.

Protein structure refinement. Crystallographic model refinement and all further electron density map calculations were carried out using the CNS program (15). All reflections in the 20.0 to $3.5 \AA$ resolution range for MVMi (PDB accession no. $1 \mathrm{MVM}$ ) (3), in the 20.0 to $3.25 \AA$ resolution range for $\mathrm{MVMp}_{\mathrm{b}}$, and in the 20 to $3.75 \AA$ resolution range for $\mathrm{MVMp}_{\mathrm{e}}$ were used during the refinement, with $5 \%$ of the data sets partitioned in a test set for monitoring the refinement process (14). Several alternating cycles of manual model rebuilding using the interactive molecular graphics program $\mathrm{O}$ (32) and refinement improved the quality of the models. The refinement protocol consisted of bulk solvent correction, geometry regularization, and least-squares conjugate-gradient refinement, followed by simulated annealing, conventional positional refinement, and individual restrained B-factor refinement. Strict NCS was applied to generate symmetry-related subunits from the coordinates of a single VP2 subunit. The MVM VP2 models were manually inspected with simulated-annealed omit maps and sigma-weighted 60 -fold averaged $2 \mathrm{~F}_{\mathrm{o}}-\mathrm{F}_{\mathrm{c}}$ and $\mathrm{F}_{\mathrm{o}}-\mathrm{F}_{\mathrm{c}}$ electron density maps and were adjusted to fit the density. N-terminal residues 1 to 38 were not built into the MVMp structures due to poor density, and residues 29 to 38 , modeled in the MVMi structure (3), remain unrefined. Water molecules were added into unassigned positive electron density (at $1.5 \sigma$ ) in difference Fourier maps that were within hydrogen bond donor or acceptor distances. The VP2 final models, residues 39 to 587, were examined for main-chain torsion angles using the PROCHECK program (35).

The MVMp $\mathrm{p}_{\mathrm{b}}, \mathrm{MVMp}_{\mathrm{e}}$, and MVMi VP2 structures were compared using the LSQ subroutine in the O program (32), the Homology program (49), and GRASP (42). Figures were generated using the programs BOBSCRIPT (22), Excel (Microsoft, Inc.), GRASP (42), Raster3D (40), and PyMol (21).

Analysis of thermal stability of virus particles. Equivalent amounts $(0.4 \mu \mathrm{g})$ of native MVMi (37), MVMp $\mathrm{p}_{\mathrm{b}}$, and MVMp $\mathrm{M}_{\mathrm{e}}$ (27) empty capsids in $40 \mu \mathrm{l}$ of $50 \mathrm{mM}$ Tris- $\mathrm{HCl}$ at $\mathrm{pH} 6.0,7.5$, or 8.8 were simultaneously heated for $10 \mathrm{~min}$ in water baths at the temperatures of $25,70,75,80$, and $85^{\circ} \mathrm{C}$ and shock frozen in dry ice. Heated samples were diluted to a final volume of $100 \mu \mathrm{l}$ with $10 \times$ phosphatebuffered saline and $\mathrm{H}_{2} \mathrm{O}$ and then assayed for hemagglutination (HA) activity with $2 \%$ mouse erythrocytes in phosphate-buffered saline $\left(2 \mathrm{~h}\right.$ at $\left.4^{\circ} \mathrm{C}\right)$ as previously described (27).

Comparative analysis of autonomous parvovirus capsid structures. The atomic models of wt full and empty CPV, CPV mutants CPV-N93R (full) and CPV-N93D (empty), FPV (empty), and PPV (empty) were obtained from the Protein Data Bank (PDB accession no. 4DPV, 2CAS, 1P5W, 1P5Y, 1C8E, and IK3V). These structures, plus the refined models of MVMi and MVMp superimposed with the least-squares subroutine in the O program (32) to obtain an overall root mean square deviation (RMSD) for their $\mathrm{C} \alpha$ positions. Differences in individual $\mathrm{C} \alpha$ positions were calculated, relative to the refined structure of $\mathrm{MVMp}_{\mathrm{b}}$, using the least-squares Homology program (49).

Protein structure accession numbers. The refined coordinates for $\mathrm{MVMp}_{\mathrm{b}}$ and MVMi VP2 have been deposited with the Protein Data Bank (PDB accession no. $1 \mathrm{Z} 14$ and $1 \mathrm{Z1C}$, respectively).

\section{RESULTS AND DISCUSSION}

MVM capsid structure. The structures of $\mathrm{MVMp}_{\mathrm{b}}$ and $\mathrm{MVMp}_{\mathrm{e}}$ have been determined to 3.25 and $3.75 \AA$ resolution, respectively. Residues 39 to 587 (the latter is the last C-terminal residue) of the VP2 capsid sequence were built into the MVMp electron density maps (Fig. 1A to G) resulting from molecular replacement and refinement procedures (Tables 1 and 2) $(15,50,59)$. The MVMp density maps were not interpretable beyond N-terminal residue 39 of the VP2 amino acid sequence. This is in contrast to the interpretation of the VP2 electron density map for the full MVMi capsid. In this DNAcontaining structure, density within the icosahedral fivefold channel was built as 10 additional residues extending from $\mathrm{N}$-terminal residue 39 , which is located directly under the fivefold channel (3). The lack of ordered density within the fivefold channel of the empty MVMp capsid is similar to observations for other empty parvovirus capsid structures $(2,55,67)$ and is consistent with the postulation that the VP2-to-VP3 cleavage that occurs in full (and not in empty) capsids and VP1 exposure of phospholipase A2 activity (23) are likely facilitated by Nterminal externalization via this channel.

The MVMp and MVMi VP2 models were refined using the CNS program $(14,15)$. The final $R_{\text {factor }}$ and $R_{\text {free }}$ were 29.85 and $30.62 \%$ for $\mathrm{MVMp}_{\mathrm{b}}, 32.35$ and $32.95 \%$ for $\mathrm{MVMp}_{\mathrm{e}}$, and 32.55 and $32.93 \%$ for MVMi (Table 2). The similarity of $R_{\text {factor }}$ and $R_{\text {free }}$ for virus structures stems from the high noncrystal- 

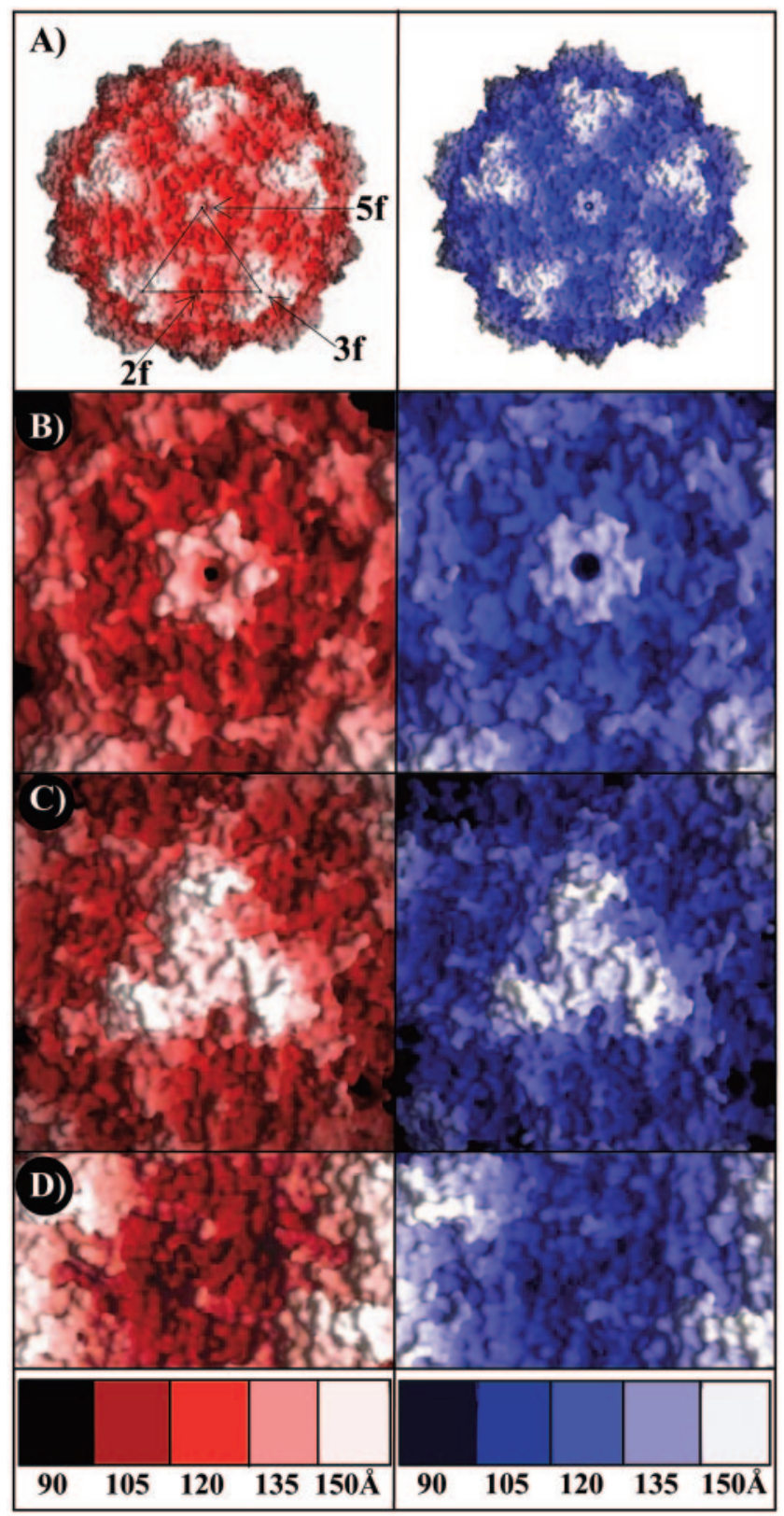

FIG. 2. Depth-cued surface representations of the MVM capsid (A) The surface topologies of $\mathrm{MVMp}_{\mathrm{b}}$ (red) and MVMi (blue) are shown with fivefold (5f), threefold (3f), and twofold (2f) axes labeled on the MVMp $\mathrm{p}_{\mathrm{b}}$ capsid. A viral asymmetric unit is depicted by a triangle bounded by two threefold axes divided by a line drawn through the twofold axis, and a fivefold axis. (B to D) Close-up views of the $\mathrm{MVMp}_{\mathrm{b}}$ (red) and MVMi (blue) capsid surfaces at the fivefold (B), threefold (C), and twofold (D) icosahedral axes. The panel at the bottom depicts the color range (in $\AA$ ) for the depth-cued distances from the viral center of the particles. This figure was generated by the GRASP program (42).

lographic icosahedral symmetry of their capsid. These $R$ values are comparable to those quoted for parvoviruses and other virus capsid structures, as detailed on the VIPER website (48). The stereochemical parameters and geometries of the model (Table 2) were consistent with those reported for other virus structures at comparable resolution.
Comparison of the electron density maps and models for the $\mathrm{MVMp}_{\mathrm{b}}$ and MVMp $\mathrm{VP} 2 \mathrm{~s}$ showed them to be superimposable (Fig. 1A and B), with an RMSD of $0.08 \AA$ for $\mathrm{C} \alpha$ atoms and $0.25 \AA$ for all 4,317 atoms (for residues 39 to 587). This observation provides structural verification that some parvovirus particles produced in a heterologous system are indistinguishable from native capsids produced in host systems (27). The results also support reports that the major capsid protein of MVM is sufficient to form native-like particles that are similar to wt particles (66). Based on the structural identity of the $\mathrm{MVMp}_{\mathrm{b}}$ and $\mathrm{MVMp} \mathrm{p}_{\mathrm{e}} \mathrm{VP} 2 \mathrm{~s}$, the higher-resolution $\mathrm{MVMp}_{\mathrm{b}}$ structure was used for all subsequent comparisons to the MVMi VP2 structure.

The MVM VP2 structures have the general parvovirus capsid viral protein topology (4). An eight-stranded $\beta$-barrel motif ( $\beta$ B to $\beta I$ ) forms the core contiguous capsid, decorated by loop insertions between the $\beta$-strands (Fig. 1K). Small stretches of antiparallel $\beta$-strands are observed in the loops between the core strands, as was reported for CPV (69). A small $\alpha$-helix $(\alpha \mathrm{A})$ spanning residues 125 to 135 that is conserved in all the parvovirus structures determined so far and lies close to the icosahedral twofold axis is also present in the MVM VP2 structure (Fig. 1K). A channel at the parvovirus icosahedral fivefold axis formed by the clustering of five symmetry-related $\beta$-ribbons (residues 153 to 171 ) between $\beta D$ and $\beta E$ (Fig. $1 \mathrm{~F}$ to $\mathrm{K}$ ) is conserved (Fig. 2A and B). A protrusion is centered at the icosahedral threefold axes (Fig. 2C), resulting from the clustering of six large surface loops, two from each threefoldsymmetry-related VP2 subunit. These loops are between $\beta E$ and $\beta F$ (residues 217 to 239) and between $\beta G$ and $\beta H$ (residues 405 to 455 ). The MVM capsid radii at the icosahedral threefold axis are $\sim 135 \AA$, while the three apexes that surround it are at $\sim 150 \AA$, leading to a slight depression at the center of the protrusions (Fig. 2A and C). A difference in the side chain conformation of surface residue E229 (torsion angles for $\mathrm{MVMp}_{\mathrm{b}} / \mathrm{MVMi}$ are as follows: $\operatorname{chi} 1=69 /-78^{\circ}$, chi $2=$ $-69 /-178^{\circ}$, and chi3 $=-54 /-54^{\circ}$ ), located at the three vertices of the threefold protrusion, results in a more "pointed" appearance in the MVMi capsid than in the MVMp capsid (Fig. 2A and C). The shoulder of the protrusion is formed by loops between $\beta B$ and $\beta C$ (residues 83 to 108 ) and between $\beta \mathrm{G}$ and $\beta \mathrm{H}$ (residues 284 to 360 ). Depressions are observed at and surrounding the icosahedral twofold axes, with capsid radii of $\sim 105 \AA$ (Fig. 2A and D), and surrounding the cylindrical structure, with a channel at icosahedral fivefold axes. The capsid radii at the icosahedral fivefold axis are $\sim 130 \AA$ (Fig. $2 \mathrm{~A}$ and B).

Structural clustering of MVMi and MVMp (MVMi/p) VP2 differences. The VP2 structures of MVMi and $M V M p_{b}$ are almost identical, with the $\mathrm{C} \alpha \mathrm{s}$ of residues 39 to 587 superimposing with an RMSD of $0.48 \AA$ (for 546 out of 549 residues) (Fig. 1K). A total of 14 amino acids (residues 10, 160, 232, 317, $321,362,366,368,388,402,410,440,455$, and 551) differ between the MVMi and MVMp VP sequences (5); all of these are within VP2. N-terminal residue 10 (glycine in MVMi and serine in MVMp) is not ordered in the current VP2 structures. At the resolution of the electron density maps calculated, the densities for the remaining 13 amino acids were consistent with the amino acid types (examples are given in Fig. 1A to $G$ ). Twelve of the 13 residues (except for residue 160, discussed 


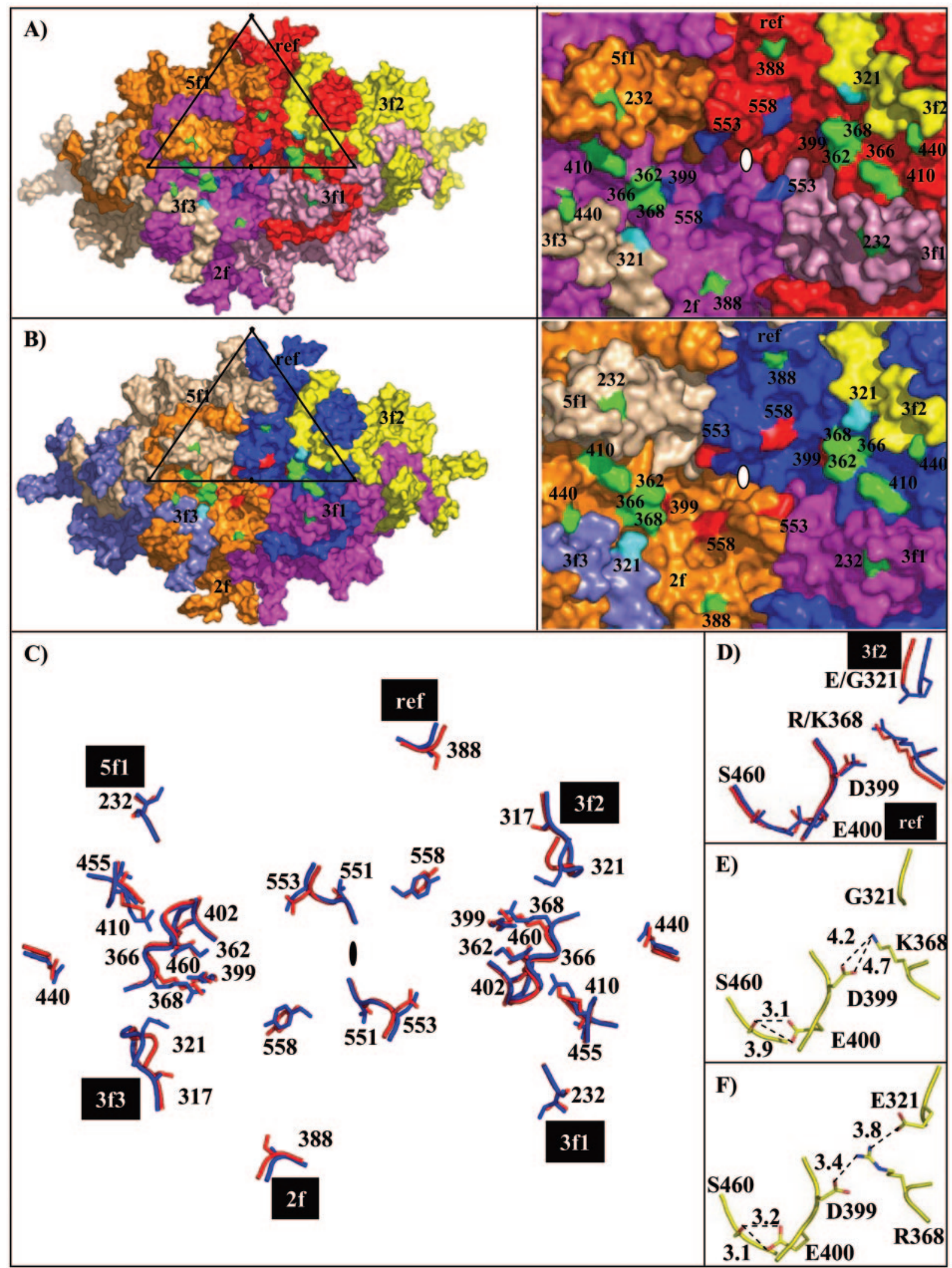

FIG. 3. Structural clustering of MVMi/p amino acid differences. (A) (Right) Surface representation of the MVMp $\mathrm{p}_{\mathrm{b}}$ capsid showing VP2 molecules related to a reference monomer (ref, in red) by icosahedral twofold ( $2 \mathrm{f}$, in pink), threefold ( $3 \mathrm{f} 1$, in purple; $3 \mathrm{f} 2$, in yellow; and $3 \mathrm{f} 3$, in salmon), and fivefold (5f, in orange) icosahedral symmetry operations. (Left) Close-up view of the MVMp $\mathrm{p}_{\mathrm{b}}$ icosahedral twofold axes, with the positions of surface MVMi/p amino acid differences colored and labeled: green for i/p differences (residues 232, 321, 362, 366, 368, 388, 410, and 


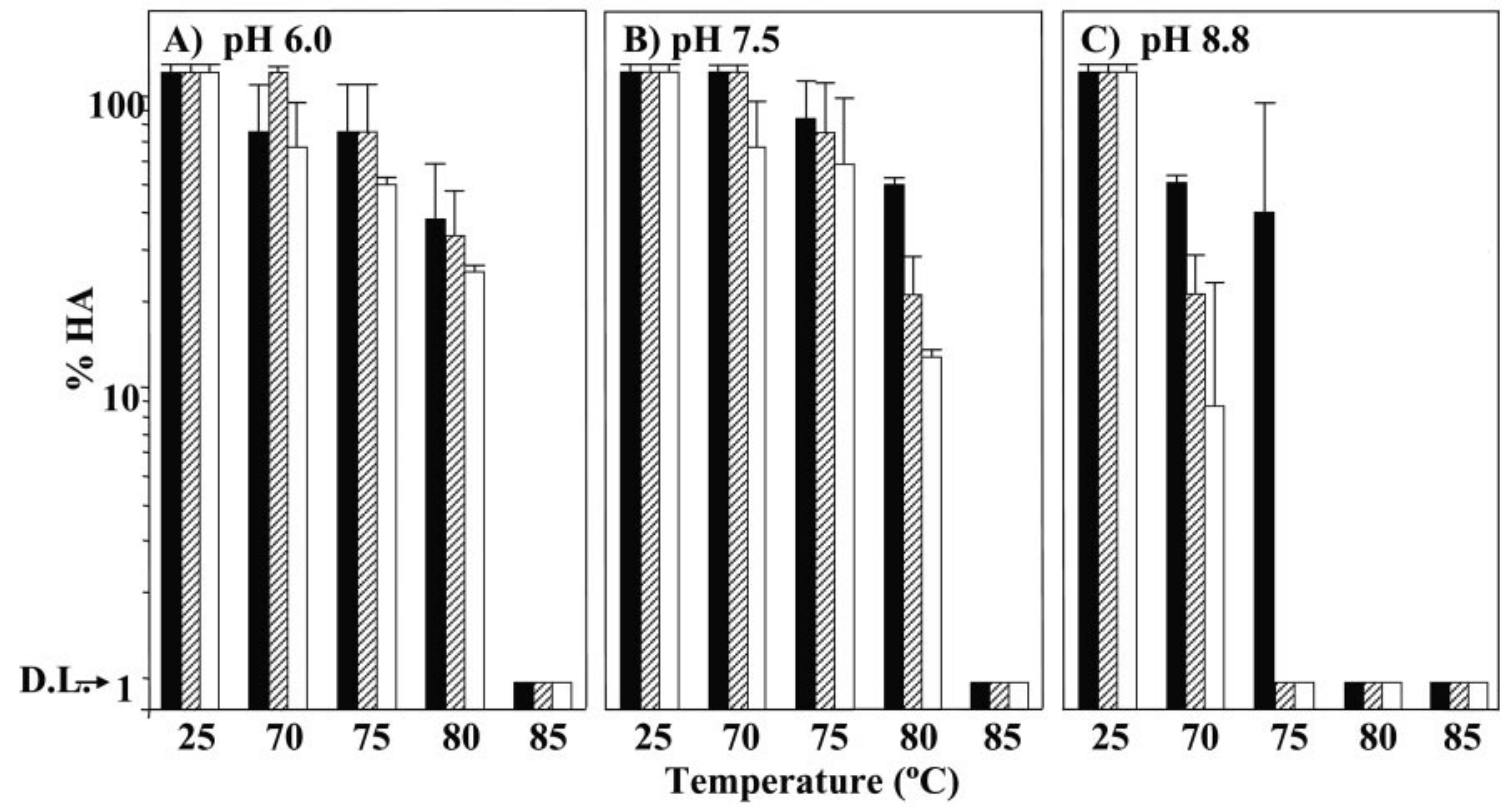

FIG. 4. MVM capsid stability. Shown are the percentages of hemagglutination activity $\left(y\right.$ axis) in mouse erythrocytes for the MVMp (open $^{\circ}$ bars) $\mathrm{MVMp}_{\mathrm{e}}$ (hatched bars), and MVMi (solid bars) capsids after incubation $(10 \mathrm{~min})$ at the different temperatures $\left(25\right.$ to $85^{\circ} \mathrm{C}, x$ axis) and the $\mathrm{pHs}$ indicated. Titers (percentage of HA normalized per microgram of capsid protein) are the reciprocal limiting dilutions causing hemagglutination. DL, detection limit. Error bars, standard errors of the means from three independent experimental runs.

below) are clustered, from symmetry-related VP monomers, in the depression at the icosahedral twofold axes, on the walls of the twofold depression, and at the shoulder of the protrusions at the icosahedral threefold axes that surround the depression (Fig. 3A and B). Eight of these 12 residues (residues 232, 321, 362, 366, 368, 388, 410, and 440) are surface exposed (Fig. 3A and B), and residue 317 is solvent accessible. Residues 399, 460,553 , and 558, which confer fibrotropism on MVMi (3), are also located in the twofold depression or on the wall surrounding it, with residues 399, 553, and 558 on the capsid surface (Fig. 3A and B). Forward mutations are selected at these four positions in the MVMi sequence when this virus, with a sitedirected mutation at allotropic residue 317 or 321 changing the amino acid type to that of MVMp, is used to infect mouse fibroblasts.

Variations in the side chains of the 12 differing MVMi/p amino acids discussed above and in the side chains of the 4 residues that are selected in forward fibrotropic MVMi mutations (but are the same in the wt viruses) result in distinct local surface topologies (Fig. 3A and B) and intersubunit contacts close to and in the depression at the icosahedral twofold axes of the MVMi and MVMp $\mathrm{b}_{\mathrm{b}}$ capsids (Fig. 3C to F). A chain of weak intra and intersubunit amino acid interactions from the wall (E321) toward the floor (R368, D399) of the twofold depression is observed in MVMi (Fig. 3F). These interactions involve $\mathrm{MVMi} / \mathrm{p}$ differing and forward mutation residues, with a weak ionic contact between R368 and E321 (allotropic residue) from a threefold-symmetry-related VP2 monomer, and a hydrogen bond between residues R368 and D399 (Fig. 3F). These interactions are not possible in $\mathrm{MVMp}_{\mathrm{b}}$, which contains G321 and K368, and the terminal oxygens of the D399 side chain are too far from the terminal amino group of K368 for a hydrogen-bonding interaction (Fig. 3E). In addition, MVMi/p differences are observed in the side chain conformations of residue E400 (immediately under the capsid surface beneath residue D399) (Fig. 3D to F). This residue is involved in hydrogen bond interactions with residue S460 (a forward fibrotropic mutant residue that is nonsurface) in the MVMi and $\mathrm{MVMp}_{\mathrm{b}}$ structures, although the interaction is slightly stronger in MVMi (Fig. 3F) than in MVMp $\mathrm{M}_{\mathrm{b}}$ (Fig. 3E). Interestingly, the

\footnotetext{
440; residue 317 is not visible in the view shown, and residue 551 is buried) and blue for forward fibrotropic mutations (residues 399,553 , and 558; residue 460 is buried under residue 399). (B) (Right) Surface representation of the MVMi capsid showing the ref (blue) and the 2f, 3f1, 3f2, 3f3, and $5 \mathrm{f} 1$ monomers (orange, pink, yellow, grey, and salmon, respectively). (Left) Close-up view of the MVMi icosahedral twofold axes, with the residues colored and labeled as in panel A, except that the forward fibrotropic residues appear red rather than blue. A viral asymmetric unit, as described in the legend to Fig. 2, is shown in panels A and B. White ovals represent approximate icosahedral twofold axes. (C) Side chain conformations of 12 of the 13 differing MVMi/p residues (residues 232, 317, 321, 362, 366, 368, 388, 402, 410, 440, 455, and 551) that are ordered within VP2 and are clustered from symmetry-related monomers. These are located at or surrounding the icosahedral twofold axes and on the shoulder of the threefold protrusions. (D to F) Close-up views of the intra- and intersubunit interactions involving allotropic and forward fibrotropic mutation residues. (D) Superimposition of the MVMp (red) and MVMi (blue) residues 321, 368, 399, 400, and 460. (E and F) Amino acids for the $\mathrm{MVMp}_{\mathrm{b}}$ and MVMi capsids, respectively, colored according to atom type (yellow, carbon; red, oxygen; blue, nitrogen), with dashed lines labeled to indicate the distance between the charged atoms that could engage in ionic (H-bond) interactions. This figure was generated with the PyMol (21) (A and B) and BOBSCRIPT (22) (C to F) programs.
} 


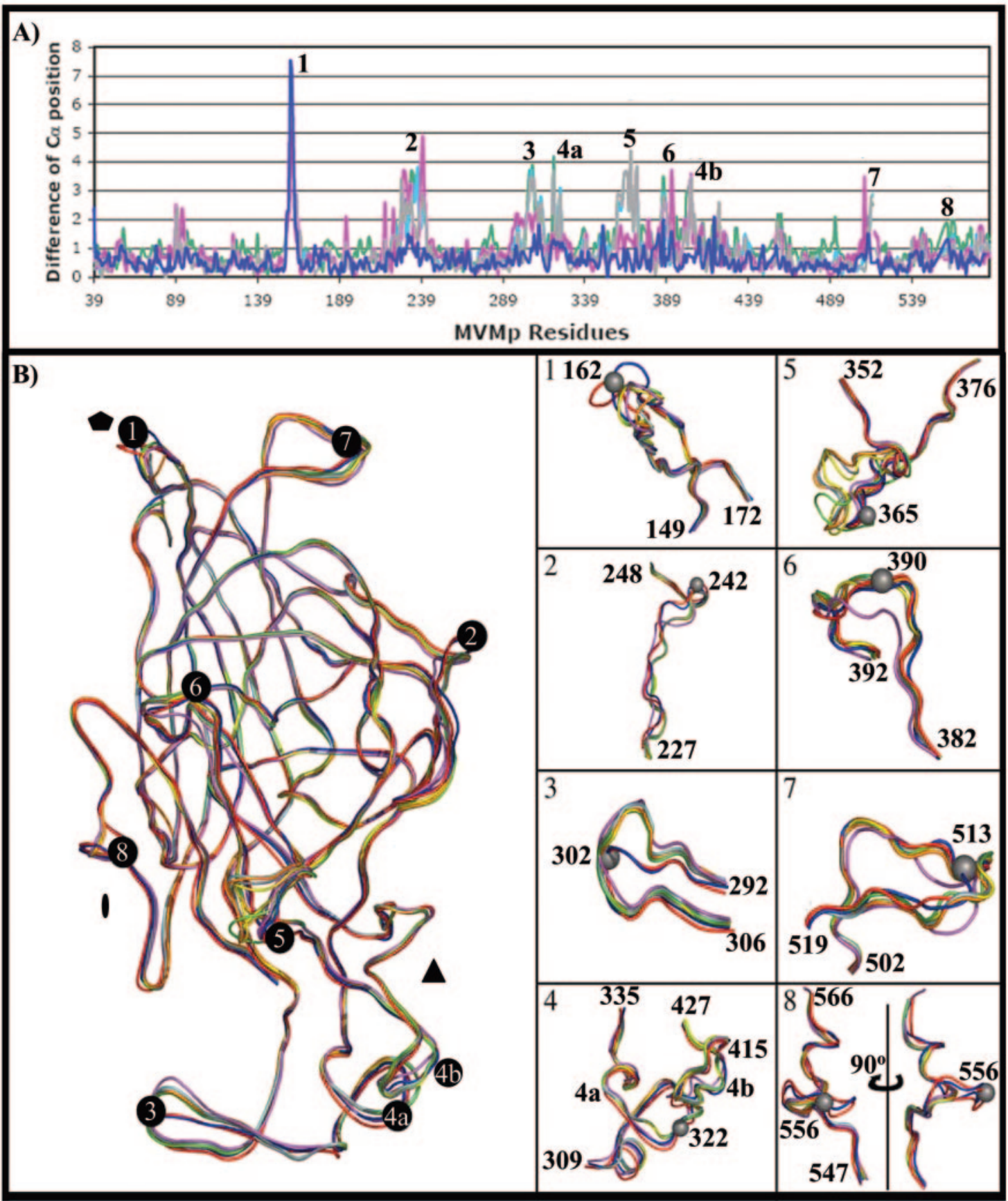

FIG. 5. Comparison of the VP2 structures of CPV, FPV, PPV, and MVM. (A) Plot of the C $\alpha$ differences between the refined VP2 structures of MVMp $p_{b}$ and MVMi (blue), CPV (wt full capsids; PDB accession no. 4DPV) (grey), CPV-N93D (mutant empty capsids; PDB accession no. 1P5Y) (cyan), FPV (wt empty capsids; PDB accession no. 1C8E) (green), and PPV (baculovirus-expressed empty capsids; PDB accession no. $1 \mathrm{~K} 3 \mathrm{~V}$ ) (pink), calculated using the Homology program (49). Regions at a $\mathrm{C} \alpha$ difference of $\geq 2.0 \AA$ between MVMp $\mathrm{p}_{\mathrm{b}}$ and MVMi are labeled 1 to 8. This figure was generated with Excel as part of the Microsoft Office package. (B) Superimposition of a coil representation of the VP2 backbone atoms of $\mathrm{MVMp}_{\mathrm{b}}$ (red), MVMi (blue), CPV (wt and mutant structures; PDB accession no. 2CAS, 4DPV, 1P5Y, and 1P5W in yellow, grey, orange, and cyan, respectively), FPV (PDB accession no. 1C8E) (green), and PPV (PDB accession no. 1K3V) (pink). Variable regions equivalent to regions 1 to 8 in panel A are labeled. The icosahedral twofold, threefold, and fivefold axes are shown as the filled oval, triangle, and pentagon, respectively. Panels on the right show close-up views of the structures at variable regions 1 to 8 . This figure was generated by PyMol (21).

site-directed/forward mutation combinations (A317T/D399G, A317T/D399A, A317T/D553N, E321G/A317T, E321G/S460A, and $\mathrm{E} 321 \mathrm{G} / \mathrm{Y} 558 \mathrm{H}$ ) that confer fibrotropism on MVMi (3) eliminate the potential for ionic interactions by the forward mutant residues (Fig. 3E and F) or alter the surface charge within the vicinity of the twofold depression. These observations and the refined $M V M p_{b}$ structure, in which the number of ionic interactions involving $\mathrm{MVMi} / \mathrm{p}$ differing residues is 
lower than that in the MVMi capsid, support previous suggestions that fibrotropism is likely controlled by a less stable MVM capsid (see also below) and/or a reduced acidic environment at or close to the icosahedral twofold axis (3). Coincidently, altered intra- and intersubunit interactions involving tropism and pathogenicity determinants are also observed in comparisons of CPV to FPV and to host range mutants with altered receptor binding properties (26). A predicted mainchain distortion in the MVMp capsid due to the allotropic amino acid difference (A317T) between MVMi and MVMp (3) was not observed in the MVMp

The remaining MVMi/p differing residue, residue 160 (of the 13 residues ordered within VP2), which is a serine in MVMi and a leucine in MVMp, is located close to the top of the loop between the $\beta$-strands making up the $\beta$-ribbons that form the fivefold channel (Fig. 1F to J). This single-amino-acid change causes a drastic conformational change in this loop region, from residue 157 to 164 , an area that was clearly interpretable in $2 \mathrm{~F}_{\mathrm{o}}-\mathrm{F}_{\mathrm{c}}$ electron density maps contoured at a $1.8 \sigma$ level (Fig. 1F and G). The different loop conformations create a topology at the top of the fivefold cylinder in $\mathrm{MVMp}_{\mathrm{b}}$ that is distinct from that in MVMi (Fig. 2A and B). The loop rearrangement increases the diameter at the top of the fivefold channel in $\mathrm{MVMp}_{\mathrm{b}}(16.7 \AA)$ relative to MVMi (8.5 ̊) (Fig. 1I and $\mathrm{J})$. This dramatic conformational rearrangement was unexpected for a serine/leucine side chain difference. There is a possibility that the topology difference is due to a capsid structural rearrangement resulting from VP2 N-terminal externalization for cleavage to VP3 in the full MVMi capsid (which would not occur in the empty $\mathrm{MVMp}_{\mathrm{b}}$ capsid) rather than to the difference in residue 160. Comparisons of other full and empty parvovirus structures have shown variation in the topology of this fivefold loop, postulated to facilitate its predicted role in enabling VP externalization $(3,23,26,63,69)$, although the differences are generally less dramatic (26). A structural study of DNA-full MVMp capsids has been initiated to enable a more complete interpretation of the observed MVMi/p fivefold differences.

MVM capsid stability. Parvovirus capsids are generally stable over wide temperature $\left(25\right.$ to $\left.70^{\circ} \mathrm{C}\right)$ and $\mathrm{pH}(\mathrm{pH} 3.0$ to 9.0$)$ ranges $(19,27)$. A comparative study of the stability of the MVM capsids was prompted by the observation that intra- and intersubunit interactions, involving clustered MVMi/p differing residues, differ between the two viruses, with MVMi predicted to be more stable. The stabilities of empty MVMi, MVMp and $\mathrm{MVMp}_{\mathrm{b}}$ capsids were measured based on their abilities to hemagglutinate mouse erythrocytes, a simple but reliable assay for MVM capsid disassembly, as supported by other techniques including tryptophan fluorescence and differential scanning calorimetry (16). Capsids were incubated at three differ-

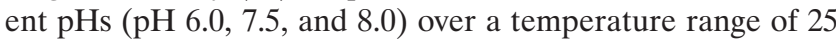
to $85^{\circ} \mathrm{C}$ (Fig. 4) and assayed. As previously described (27), both MVMp capsids were equally stable during the assay, with complete disassembly occurring only at high temperatures, although the $\mathrm{MVMp}_{\mathrm{b}}$ capsids consistently showed slightly lower HA activity. The MVMi capsid, however, was clearly more stable than both types of MVMp capsids at any pH (Fig. 4A to C). In general, MVM capsids were less stable at increasing $\mathrm{pHs}$, and the differences between the strains were more pronounced at $\mathrm{pH} 8.8$ (Fig. 4C). At this $\mathrm{pH}$ and temperatures of $\geq 70^{\circ} \mathrm{C}$, the stability of MVMp capsids was dramatically lower than that of the MVMi capsid. Both MVMp capsids were completely disassembled by $75^{\circ} \mathrm{C}$ at this $\mathrm{pH}$ (Fig. 4C), while the MVMi capsid still retained approximately $50 \%$ of the activity observed at the lower $\mathrm{pHs}$ at $80^{\circ} \mathrm{C}$. Complete disassembly of the MVMi capsid at $\mathrm{pH} 8.8$ occurred at $80^{\circ} \mathrm{C}$, rather than the $85^{\circ} \mathrm{C}$ observed at $\mathrm{pHs} 6.0$ and 7.5 .

The calculated pI (25) for both the MVMi and MVMp VP2 amino acid sequences is $\sim 5.8$, and the overall charge of both capsids should be the same at the $\mathrm{pHs}$ tested. The $\mathrm{pK}_{\mathrm{a}}$ values of the terminal amide groups of the lysine and arginine MVMp/i difference at amino acid position 368 are $\sim 10$ and $\sim 12$, respectively. These amide groups will be positively charged at pH 6.0 to 8.8. K368 of MVMp is too far from D399 to engage in a salt bridge interaction (Fig. 3E). Thus, at the higher $\mathrm{pH}, 8.8$, the interactions of $\mathrm{R} 368$ with the adjacent D399 and E321 (Fig. 3F) will result in a more stable capsid for MVMi than for MVMp, which contains a glycine at amino acid position 321 and a lysine at position 368. Therefore, the differential capsid stability observed between MVMi and MVMp could be due to the difference in local surface interactions across the twofold axes (Fig. 3C to F).

Comparison of MVM capsid structures to those of other autonomous parvoviruses. The MVM VP2 structure topology is very similar to those available for the autonomous parvoviruses CPV, FPV, and PPV (Fig. 5A and B). The structures are superimposable (Fig. 5B), with an overall RMSD of 0.4 to 0.6 $\AA$ between structurally equivalent VP2 C $\alpha$ atoms. These values are within the range calculated for the superimposition of MVMp $_{\mathrm{b}}$ and MVMi VP2 (0.48 $\left.\mathrm{A}\right)$, wt CPV and wt FPV, or wt CPV and CPV mutant VP2 structures (26). However, there are surface loop regions (labeled 1 to 8 in Fig. 5A and B, Fig. 6A, and Table 3 ) that show $\mathrm{C} \alpha$ differences of as much as $\sim 5 \AA$ (Fig. $5 \mathrm{~A})$. These regions also differ structurally between highly homologous parvovirus strains, such as wt CPV and wt FPV, wt CPV and its host range mutants, and also MVMi and MVMp $(2,26,53,61)$. These local loop differences cluster, from icosahedral-symmetry-related monomers, to create local variations on the characteristic parvovirus capsid features at the fivefold axes, at the shoulder of the threefold axes, and at and surrounding the twofold axes (Fig. 3B and D and 6A). An analogous comparison of dependovirus VP3 structures and 3-dimensional models also identified similar variable regions on their capsids that are clustered from symmetry-related monomers (44). The dependovirus differences are postulated to control receptor recognition and antigenic phenotypes. The suggested functional roles of the variable autonomous virus capsid regions are discussed below.

Role of common variable parvovirus capsid surface loop regions in viral tropism and pathogenicity. The role of variable region 7 , a loop located between $\beta \mathrm{H}$ and $\beta \mathrm{I}$, is currently unknown. Variable region 1 contains MVM residue 160 and, as discussed above, structural rearrangements of this capsid region are likely involved in the externalization of VP1 and VP2 (Table 3), for phospholipase A2 function and VP2-to-VP3 cleavage, respectively. Variable regions 2 and 3 contain CPV and FPV residues that form part of two major antigenic epitopes (57). Residues in the four remaining variable regions (variable regions 4, 5, 6, and 8), plus region 3 (Fig. 5 and 6A), have been mapped previously as being important for parvovi- 


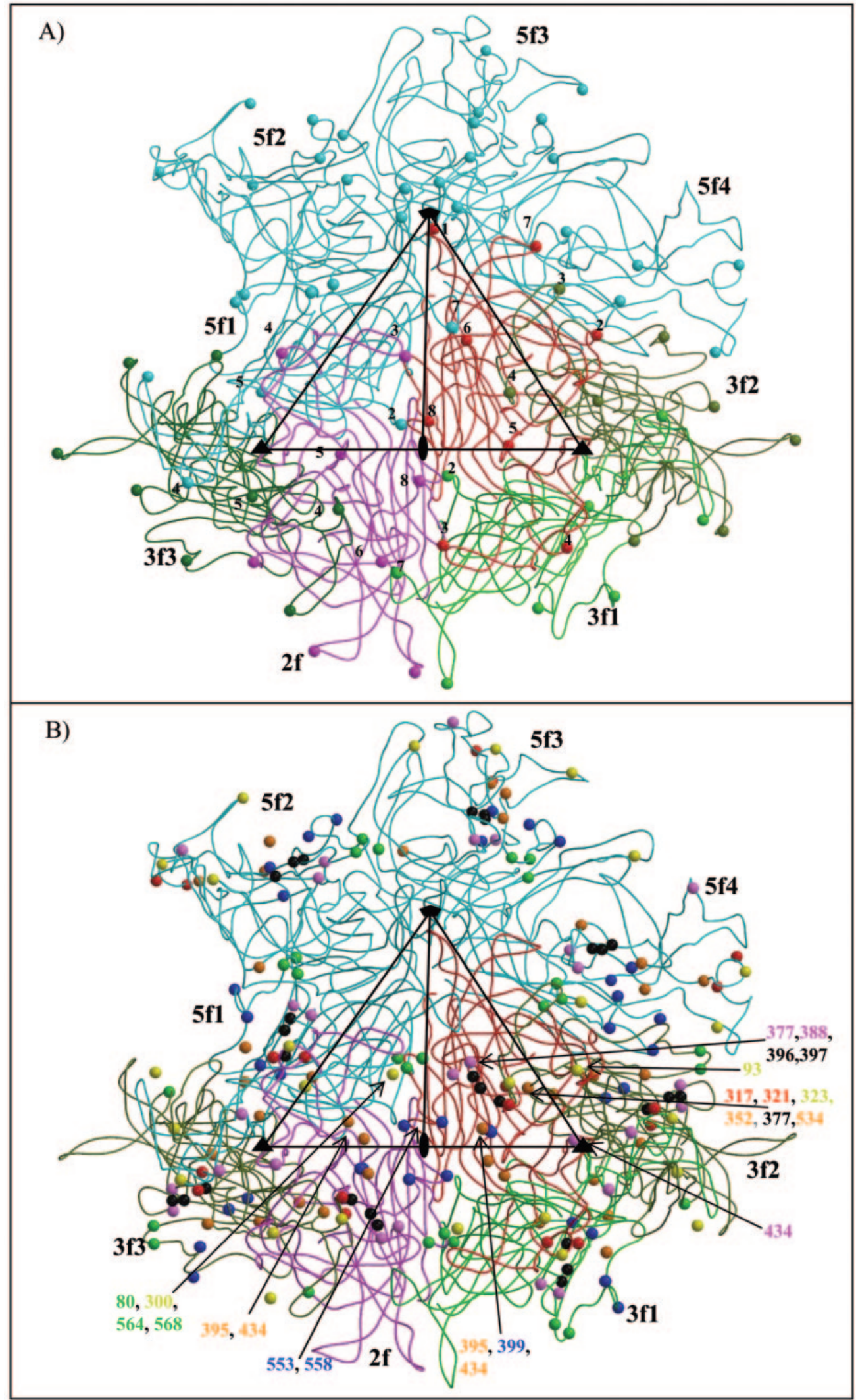

FIG. 6. Correlation of variable parvovirus capsid surface regions with tropism and pathogenicity determinants. (A) Variable regions 1 to 8

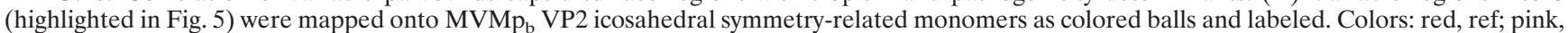
twofold (2f); light to dark green, threefold ( $3 \mathrm{f} 1$ to $3 \mathrm{f} 3$ ); cyan, fivefold (5f1 to $5 \mathrm{f} 4$ ). (B) The C $\alpha$ positions of residues implicated in tropism and pathogenicity determination for MVM, CPV/FPV, PPV, and AMDV are shown as colored balls mapped to MVMp $\mathrm{p}_{\mathrm{b}}$ V icosahedral symmetry- 
TABLE 3. Locations of variable regions on parvovirus capsids and suggested functional roles

\begin{tabular}{|c|c|c|c|}
\hline Region & Capsid location ${ }^{a}$ & Functional role & Reference(s) \\
\hline 1 & Top of fivefold axes & VP1 and VP2 externalization & $3,23,26,63,69$ \\
\hline 2 & Shoulder of threefold protrusion & CPV/FPV antigenicity & 57 \\
\hline 3 & Wall between two- and fivefold axes & $\begin{array}{l}\text { CPV/FPV tissue tropism/pathogenicity/receptor } \\
\text { attachment and antigenicity }\end{array}$ & $17,26,31,45,57,61,62$ \\
\hline \multirow[t]{2}{*}{4} & \multirow[t]{2}{*}{ Shoulder of threefold protrusion } & $\begin{array}{l}\text { MVM allotropic determinant } \\
\text { CPV/FPV tissue tropism/pathogenicity, receptor } \\
\text { attachments and antigenicity }\end{array}$ & $\begin{array}{l}6 \\
17,26,30,45,60-62\end{array}$ \\
\hline & & AMDV tissue tropism/pathogenicity & 24,39 \\
\hline \multirow[t]{2}{*}{5} & \multirow[t]{2}{*}{ Wall of twofold depression } & $\begin{array}{l}\text { MVM fibrotropism } \\
\text { Controls pH-dependent HA of CPV and FPV, and } \\
\text { sialic acid binding }\end{array}$ & $7,53,60$ \\
\hline & & AMDV tissue tropism/pathogenicity & 24,39 \\
\hline \multirow[t]{2}{*}{6} & \multirow[t]{2}{*}{ Wall between two- and fivefold axes } & $\begin{array}{l}\text { CPV/FPV tissue tropism/pathogenicity and receptor } \\
\text { attachment }\end{array}$ & 26,45 \\
\hline & & PPV tropism/pathogenicity & 55 \\
\hline 7 & $\begin{array}{l}\text { Floor of depression surrounding } \\
\text { fivefold axes }\end{array}$ & Unknown & \\
\hline 8 & Floor of twofold depression & MVM fibrotropism and receptor attachment & $\begin{array}{l}\text { 2; López-Bueno et al, } \\
\text { unpublished data }\end{array}$ \\
\hline
\end{tabular}

${ }^{a}$ See, e.g., Fig. 6A.

rus tropism, pathogenicity determination, hemagglutination behavior, or receptor attachment (Table 3). The amino acid residues involved in controlling these functions for AMDV, CPV, FPV, MVM, and PPV are mapped onto homologous capsid regions of the $\mathrm{MVMp}_{\mathrm{b}}$ structure in Fig. $6 \mathrm{~B}$.

The clustering of $\mathrm{MVMi} / \mathrm{p}$ allotropic residues (A317T, E321G) (region 4 in Fig. 5B and 6A; red balls in Fig. 6B) and of residues involved in MVMi forward fibrotropic mutations (residues 399, 460, 553, and 558) (regions 5 and 8 in Fig. 5B and $6 \mathrm{~A}$; blue balls in Fig. 6B) suggests a vital role for this capsid region in $\mathrm{MVMi} / \mathrm{p}$ in cellular recognition. $\mathrm{CPV} / \mathrm{FPV}$ residue 323 , which plays a role in tissue tropism and pathogenicity and is postulated to form part of the receptor attachment $(26,30)$, is structurally close to MVM's allotropic residue 321 (Fig. 6B), suggesting possible utilization of common capsid regions for host cell recognition. For AMDV, VP2 residues 352, 395, 434, and 534 (orange balls in Fig. 6B), implicated in tropism and pathogenicity determinations (39), are mapped to variable regions 4 and 5 in Fig. 5B and 6A. The pathogenicity determinants for PPV involve two VP2 amino acids, residues 377 and 388 (55) (pink balls in Fig. 6B), which map close to region 6 in Fig. 5B and 6A on the wall between the twofold and fivefold axes. A third PPV residue, residue 434 (close to the icosahedral threefold axes [Fig. 6B]), previously implicated as a pathogenicity determinant, has been shown to be less impor- tant (P. Tijssen, personal communication). The role of receptor recognition in AMDV and PPV tropism and pathogenicity determination requires further investigation, but surface clustering of the determinant residues in analogous structural positions in the CPV/FPV and MVMi/p model systems suggests a commonality in functionality.

Although the primary cell surface receptor utilized for infection by MVM is not known, treatment of susceptible cells with neuraminidase, which removes terminal sialic acids, abolishes MVM reinfection, identifying this carbohydrate as a component of the cell surface receptor (20; A. López-Bueno, M. P. Rubio, N, Bryant, R. McKenna, M. Agbandje-McKenna, and J. M. Almendral, unpublished data). Interestingly, CPV/FPV $\mathrm{HA}$, which involves sialic acid recognition, is dictated by CPV residues R377, E396, and $\mathrm{R} 397$ (60) on the wall of the twofold dimple (regions 4 and 6 in Fig. 6A; black balls in Fig. 6B), with R377 being structurally proximate to MVM residues 317 and 321 (Fig. 6B). The sialic acid binding site for the MVMp capsid, leading to infection, was recently mapped to the icosahedral twofold axes, highlighting the role that receptor recognition, involving differing residues, plays in MVM pathogenesis (López-Bueno et al., unpublished data). In addition, the correlation of slight structural alterations within or close to the icosahedral twofold depression, due to disruption of intra- and intersubunit amino acid interactions (Fig. 3E and F), with

related monomers. Ball colors: red, MVM allotropic residues (317 and 321); blue, MVMi forward mutations conferring fibrotropism (residues 399 553, and 558); yellow, CPV tropism and pathogenicity determinants, and proposed receptor attachment residues (residues 93, 300, and 323); black, CPV sialic acid binding residues (residues 377, 396, and 397); green, FPV tropism and pathogenicity determinants (residues 80, 564, and 568); pink, PPV tropism and pathogenicity determinants (residues 377, 388, and 434); orange, AMDV tropism and pathogenicity determinants (residues 352 , 395,434 , and 534). The viral asymmetry unit, as defined in Fig. 2A, is shown in both panels. Both panels are shown approximately down the icosahedral twofold axes. This figure was generated by the BOBSCRIPT program (22). 
mutations that change MVM tropism in vitro likely illustrates the nature of the tight regulations required for successful viruscell interaction during MVM infection. It is also likely that MVM HA involves the clustered MVMi/p differing residues at the icosahedral twofold axes. Thus, the alteration of the contacts/interactions of the capsid amino acids with sialic acid could lead to the differential MVMi/p capsid HA activity/stability observed (Fig. 4).

In conclusion, the available high-resolution structures of CPV, FPV, and PPV and those reported here for MVM, in addition to a pseudo-atomic model available for AMDV-G, provide a means to attempt the correlation of the vast amount of biology data available on parvovirus tropism and pathogenicity to the capsid structure. This analysis indicates that tropism and pathogenicity determination for highly homologous parvovirus strains is likely a precisely regulated phenotype dictated by minor changes on the capsid surface, mostly located close to or surrounding the icosahedral twofold axes (Fig. 6A and $\mathrm{B}$ ). The mapping of a binding site for sialic acid, a component of the productive receptor, in this region of the MVMp capsid (López-Bueno et al., unpublished results) and the colocalization of CPV/FPV tropism and pathogenicity determinants with a proposed receptor attachment footprint (26) warrant reexamination of the roles that receptors and intracellular factors $(29,56)$ play in parvovirus host range determination.

\section{ACKNOWLEDGMENTS}

We are grateful to Jean Rommelaere (DKFZ, Heidelberg, Germany) and Peter Tattersall (Yale University, New Haven, CT) for providing the MVM strains. We also thank the staff at DSRS (United Kingdom) and DESY (Germany) for help during synchrotron data collection. The experimental support of Beatriz Maroto and Noelia Valle in the hemagglutination assays is also acknowledged.

This study was supported by the National Science Foundation (MCB 0212846, to M.A.-M.), CSIC-British Council (Integrated Action, HB1998-0173 to C.F.-F. and 1999/2000-8125 to M.A.-M.), and Comunidad Autónoma de Madrid (ref. 07B/0020/2002, to J.M.A.).

\section{REFERENCES}

1. Agbandje, M., S. Kajigaya, R. McKenna, N. S. Young, and M. G. Rossmann. 1994. The structure of human parvovirus B19 at $8 \AA$ resolution. Virology 203:106-115.

2. Agbandje, M., R. McKenna, M. G. Rossmann, M. L. Strassheim, and P. R. Parrish. 1993. Structure determination of feline panleukopenia virus empty particles. Proteins 16:155-171.

3. Agbandje-McKenna, M., A. L. Llamas-Saiz, F. Wang, P. Tattersall, and M. G. Rossmann. 1998. Functional implications of the structure of the murine parvovirus, minute virus of mice. Structure 6:1369-1381.

4. Agbandje-McKenna, M., C. R. Parrish, and M. G. Rossmann. 1995. The structure of parvoviruses. Semin. Virol. 6:299-309.

5. Astell, C. R., E. M. Gardiner, and P. Tattersall. 1986. DNA sequence of the lymphotropic variant of minute virus of mice, MVM(i), and comparison with the DNA sequence of the fibrotropic prototype strain. J. Virol. 57:656-669.

6. Ball-Goodrich, L. J., and P. Tattersall. 1992. Two amino acid substitutions within the capsid are coordinately required for acquisition of fibrotropism by the lymphotropic strain of minute virus of mice. J. Virol. 66:3415-3423.

7. Barbis, D. P., S.-F. Chang, and C. R. Parrish. 1992. Mutations adjacent to the dimple of the canine parvovirus capsid structure affect sialic acid binding. Virology 191:301-308.

8. Bergeron, J., B. Hébert, and P. Tijssen. 1996. Genome organization of the Kresse strain of porcine parvovirus: identification of allotropic determinant and comparison with those of NADL-2 and field isolates. J. Virol. 70:25082515.

9. Bloom, M. E., J. M. Fox, B. D. Berry, K. L. Oie, and J. B. Wolfinbarger. 1998. Construction of pathogenic molecular clones of Aleutian mink disease parvovirus that replicate both in vivo and in vitro. Virology 251:288-296.

10. Bloom, M. E., H. Kanno, S. Mori, and J. B. Wolfinbarger. 1994. Aleutian mink disease parvovirus: puzzles and paradigms. Infect. Agents Dis. 3:279301.

11. Brownstein, D. G., A. L. Smith, R. O. Jacoby, E. A. Johnson, G. Hansen, and
P. Tattersall. 1991. Pathogenesis of infection with a virulent allotropic variant of minute virus of mice and regulation by host genotype. Lab. Investig. 65:357-363.

12. Brownstein, D. G., A. L. Smith, E. A. Johnson, D. J. Pintel, L. K. Naeger, and P. Tattersall. 1992. The pathogenesis of infection with minute virus of mice depends on expression of the small nonstructural protein NS2 and on the genotype of the allotropic determinants VP1 and VP2. J. Virol. 66:31183124 .

13. Bruemmer, A., F. Scholari, M. Lopez-Ferber, J. F. Conway, and E. A. Hewat. 2005. Structure of an insect parvovirus (Junonia coenia Densovirus) determined by cryo-electron microscopy. J. Mol. Biol. 347:791-801.

14. Brunger, A. T. 1992 . The free $R$ value: a novel statistical quantity for assessing the accuracy of crystal structures. Nature 355:472-474.

15. Brunger, A. T., P. D. Adams, G. M. Clore, W. L. DeLano, P. Gros, R. W. Grosse-Kunstleve, J. S. Jiang, J. Kuszewski, M. Nilges, N. S. Pannu, R. J. Read, L. M. Rice, T. Simonson, and G. L. Warren. 1998. Crystallography and NMR system: a new software suite for macromolecular structure determination. Acta Crystallogr. D 54:905-921.

16. Carreira, A., M. Menéndez, J. Reguera, J. M. Almendral, and M. G. Mateu. 2004. In vitro disassembly of a parvovirus capsid and effect on capsid stability of heterologous peptide insertions in surface loops. J. Biol. Chem. 279:65176525 .

17. Chang, S.-F., J. Y. Sgro, and C. R. Parrish. 1992. Multiple amino acids in the capsid structure of canine parvovirus coordinately determine the canine host range and specific antigenic and hemagglutination properties. J. Virol. 66: 6858-6867.

18. Chipman, P. R., M. Agbandje-McKenna, S. Kajigaya, K. E. Brown, N. S. Young, T. S. Baker, and M. G. Rossmann. 1996. Cryo-electron microscopy studies of empty capsids of human parvovirus B19 complexed with its cellular receptor. Proc. Natl. Acad. Sci. USA 93:7502-7506.

19. Cotmore, S. F., A. M. D'abramo, Jr., C. M. Ticknor, and P. Tattersall. 1999. Controlled conformational transitions in the MVM virion expose the VP1 $\mathrm{N}$-terminus and viral genome without particle disassembly. Virology 254: 169-181.

20. Cotmore, S. F., and P. Tattersall. 1987. The autonomously replicating parvoviruses of vertebrates. Adv. Virus Res. 33:91-174.

21. DeLano, W. L. 2002. The PyMol molecular graphics systems. DeLano Scientific, San Carlos, CA.

22. Esnouf, R. 1999. BOBSCRIPT. Further additions to MOLSCRIPT version 1.4 , including reading and contouring of electron-density maps. Acta Crystallogr. D 55:938-940.

23. Farr, G. A., and P. Tattersall. 2004. A conserved leucine that constricts the pore through the capsid fivefold cylinder plays a central role in parvoviral infection. Virology 323:243-256.

24. Fox, J. M., M. A. McCrackin Stevenson, and M. E. Bloom. 1999. Replication of Aleutian mink disease parvovirus in vivo is influenced by residues in the VP2 protein. J. Virol. 73:8713-8719.

25. Gasteiger, E., A. Gattiker, C. Hoogland, I. Ivanyi, R. D. Appel, and A. Bairoch. 2003. ExPASy: the proteomics server for in-depth protein knowledge and analysis. Nucleic Acids Res. 31:3784-3788.

26. Govindasamy, L., K. Hueffer, C. R. Parrish, and M. Agbandje-McKenna. 2003. Structures of host range-controlling regions of the capsids of canine and feline parvoviruses and mutants. J. Virol. 77:12211-12221.

27. Hernando, E., A. L. Llamas-Saiz, C. Foces-Foces, R. McKenna, I. Portman, M. Agbandje-McKenna, and J. M. Almendral. 2000. Biochemical and physical characterization of parvovirus minute virus of mice virus-like particles. Virology 267:299-309.

28. Horiuchi, M., H. Goto, N. Ishiguro, and M. Shinagawa. 1994. Mapping of determinants of the host range for canine cells in the genome of canine parvovirus using canine parvovirus/mink enteritis virus chimeric viruses. J. Gen. Virol. 75:1319-1328.

29. Horiuchi, M., N. Ishiguro, H. Goto, and M. Shinagawa. 1992. Characterization of the stage(s) in the virus replication cycle at which the host-cel specificity of the feline parvovirus subgroup is regulated in canine cells Virology 189:600-608

30. Hueffer, K., L. Govindasamy, M. Agbandje-McKenna, and C. R. Parrish. 2003. Combinations of two capsid regions control host range and specific transferrin receptor binding by canine parvovirus. J. Virol. 77:10099-10105.

31. Hueffer, K., J. S. Parker, W. S. Weichert, R. E. Geisel, J.-Y. Sgro, and C. R. Parrish. 2003. The natural host range shift and subsequent evolution of canine parvovirus resulted from virus-specific binding to the canine transferrin receptor. J. Virol. 77:1718-1726.

32. Jones, T., J.-Y. Zou, S. W. Cowan, and M. Kjeldgaard. 1991. Improved methods for building protein models in electron density maps and the location of errors in these models. Acta Crystallogr. A 97:110-119.

33. Kaufmann, B., A. A. Simpson, and M. G. Rossmann. 2004. The structure of human parvovirus B19. Proc. Natl. Acad. Sci. USA 101:11628-11633.

34. Kronenberg, S., J. A. Kleinschmidt, and B. Böttcher. 2001. Electron cryomicroscopy and image reconstruction of adeno-associated virus type 2 empty capsids. EMBO Rep. 2:997-1002.

35. Laskowski, R. A., M. W. MacArthur, D. S. Moss, and J. M. Thornton. 1993. 
PROCHECK: a program to check the stereochemical quality of protein structures. J. Appl. Crystallogr. 26:283-291.

36. Llamas-Saiz, A. L., M. Agbandje-McKenna, J. S. L. Parker, A. T. M. Wahid, C. R. Parrish, and M. G. Rossmann. 1996. Structural analysis of a mutation in canine parvovirus which controls antigenicity and host range. Virology 225:65-71.

37. Llamas-Saiz, A. L., M. Agbandje-McKenna, W. R. Wikoff, J. Bratton, P. Tattersall, and M. G. Rossmann. 1997. Structure determination of minute virus of mice. Acta Crystallogr. D 53:93-102.

38. Maxwell, I. H., A. L. Spitzer, F. Maxwell, and D. J. Pintel. 1995. The capsid determinant of fibrotropism for the MVMp strain of minute virus of mice functions via VP2 and not VP1. J. Virol. 69:5829-5832.

39. McKenna, R., N. H. Olson, P. R. Chipman, T. S. Baker, T. F. Booth, J. Christensen, B. Aasted, J. M. Fox, M. E. Bloom, J. B. Wolfinbarger, and M. Agbandje-McKenna. 1999. Three-dimensional structure of Aleutian mink disease parvovirus: implications for disease pathogenicity. J. Virol. 73:6882-6891.

40. Merritt, E. A., and D. J. Bacon. 1997. Raster3D—photorealistic molecular graphics. Methods Enzymol. 277:505-524.

41. Molitor, T. W., and H. S. Joo. 1990. Clinical and pathological features of porcine parvovirus-related disease and its diagnosis, p. 135-150. In P. Tijssen (ed.), Handbook of parvoviruses, vol. II. CRC Press, Boca Raton, Fla.

42. Nicholls, A., K. A. Sharp, and B. Honig. 1991. Protein folding and association: insights from the interfacial and thermodynamic properties of hydrocarbons. Proteins 11:281-296.

43. Otwinowski, Z., and W. Minor. 1997. Processing X-ray diffraction data collected in oscillation mode. Methods Enzymol. 276:307-326.

44. Padron, E., V. D. Bowman, N. Kaludov, L. Govindasamy, H. Levy, P. Nick, R. McKenna, N. Muzyczka, J. A. Chiorini, T. S. Baker, and M. AgbandjeMcKenna. 2005. The structure of adeno-associated virus 4. J. Virol. 79:50475058.

45. Parker, J. S. L., and C. R. Parrish. 1997. Canine parvovirus host range is determined by the specific conformation of an additional region of the capsid. J. Virol. 71:9214-9222.

46. Parrish, C. R., C. F. Aquadro, and L. E. Carmichael. 1988. Canine host range and a specific epitope map along with variant sequences in the capsid protein gene of canine parvovirus and related feline, mink and raccoon parvoviruses. Virology 166:293-307.

47. Ramírez, J. C., A. Fiaren, and J. M. Almendral. 1996. Parvovirus minute virus of mice strain I multiplication and pathogenesis in the newborn mouse brain are restricted to proliferative areas and to migratory cerebral young neurons. J. Virol. 70:8109-8116.

48. Reddy, V. S., P. Natarajan, B. Okerberg, K. Li, K. V. Damodaran, R. T. Morton, C. L. Brooks III, and J. E. Johnson. 2001. Virus Particle Explorer (VIPER), a website for virus capsid structures and their computational analyses. J. Virol. 75:11943-11947.

49. Rossmann, M. G., and P. Argos. 1976. Exploring structural homology of proteins. J. Mol. Biol. 105:75-95.

50. Rossmann, M. G., R. McKenna, L. Tong, D. Xia, J. Dai, H. Wu, H. K. Choi, and R. E. Lynch. 1992. Molecular replacement real-space averaging. J. Appl. Crystallogr. 25:166-180.

51. Segovia, J. C., J. A. Bueren, and J. M. Almendral. 1995. Myeloid depression follows infection of susceptible newborn mice with the parvovirus minute virus of mice (strain i). J. Virol. 69:3229-3232.

52. Segovia, J. C., J. M. Gallego, J. A. Bueren, and J. M. Almendral. 1999. Severe leukopenia and dysregulated erythropoiesis in SCID mice persistently infected with the parvovirus minute virus of mice. J. Virol. 73:1774-1784.

53. Simpson, A. A., V. Chandrasekar, B. Hebert, G. M. Sullivan, M. G. Rossmann, and C. R. Parrish. 2000. Host range and variability of calcium binding by surface loops in the capsids of canine and feline parvoviruses. J. Mol. Biol. 300:597-610.

54. Simpson, A. A., P. R. Chipman, T. S. Baker, P. Tijssen, and M. G. Rossmann. 1998. The structure of an insect parvovirus (Galleria mellonella densovirus) at $3.7 \AA$ resolution. Structure 6:1355-1367.

55. Simpson, A. A., B. Hébert, G. M. Sullivan, C. R. Parrish, Z. Zádori, P. Tijssen, and M. G. Rossmann. 2002. The structure of the porcine parvovirus: comparison with related viruses. J. Mol. Biol. 315:1189-1198.

56. Spalhotz, B. A., and P. Tattersall. 1983. Interaction of minute virus of mice with differentiated cells: strain-dependent target cell specificity is mediated by intracellular factors. J. Virol. 46:937-939.

57. Strassheim, L. S., A. Gruenberg, P. Veijalainen, J.-Y. Sgro, and C. R. Parrish. 1994. Two dominant neutralizing antigenic determinants of canine parvovirus are found on the threefold spike of the virus capsid. Virology 198:175-184.

58. Tattersall, P., A. J. Shatkin, and P. Ward. 1977. Sequence homology between the structural polypeptides of minute virus of mice. J. Mol. Biol. 111:375-394.

59. Tong, L., and M. G. Rossmann. 1997. Rotation function calculations with GLRF program. Methods Enzymol. 276:594-611.

60. Tresnan, D. B., L. Southard, W. Weichert, J.-Y. Sgro, and C. R. Parrish. 1995. Analysis of the cell and erythrocyte binding activities of the dimple and canyon regions of the canine parvovirus capsid. Virology 211:123-132.

61. Truyen, U., M. Agbandje, and C. R. Parrish. 1994. Characterization of the feline host range and a specific epitope of feline panleukopenia virus. Virology 200:494-503.

62. Truyen, U., J. F. Evermann, E. Vieler, and C. R. Parrish. 1996. Evolution of canine parvovirus involved loss and gain of feline host range. Virology 215:186-189.

63. Tsao, T., M. S. Chapman, M. Agbandje, W. Keller, K. Smith, H. Wu, M. Luo, T. J. Smith, M. G. Rossmann, R. W. Compans, and C. R. Parrish. 1991. The three-dimensional structure of canine parvovirus and its functional implications. Science 251:1456-1464.

64. Walters, R., M. Agbandje-McKenna, V. D. Bowman, T. O. Moninger, N. H. Olson, M. Seiler, J. A. Chiorini, T. S. Baker, and J. Zabner. 2004. Structure of adeno-associated virus serotype 5. J. Virol. 78:3361-3371.

65. Wikoff, W. R., G. Wang, C. R. Parrish, R. H. Cheng, M. L. Strassheim, T. S. Baker, and M. G. Rossmann. 1994. The structure of a neutralized virus: canine parvovirus complexed with neutralizing antibody fragment. Structure 15:595-607.

66. Willwand, K., and B. Hirt. 1993. The major capsid protein VP2 of minute virus of mice (MVM) can form particles which bind to the $3^{\prime}$-terminal hairpin of MVM replicative-form DNA and package single-stranded viral progeny DNA. J. Virol. 67:5660-5663.

67. Wu, H., W. Keller, and M. G. Rossmann. 1993. Determination and refinement of the canine parvovirus empty-capsid structure. Acta Crystallogr. D 49:572-579.

68. Xie, Q., W. Bu, S. Bhatia, J. Hare, T. Somasundaram, A. Azzi, and M. S. Chapman. 2002. The atomic structure of adeno-associated virus (AAV-2), a vector for human gene therapy. Proc. Natl. Acad. Sci. USA 99:10405-10410.

69. Xie, Q., and M. S. Chapman. 1996. Canine parvovirus capsid structure, analyzed at $2.9 \AA$ A resolution. J. Mol. Biol. 264:497-520. 